\title{
Functional anatomy of the nasal bones and adjacent structures. Consequences for nasal surgery*
}

\author{
Mariola Popko', Suzanne A.M.W. Verlinde-Schellekens², Egbert H. Huizing³, \\ Ronald L.A.W. Bleys ${ }^{2}$ \\ Rhinology 56: 89-95, 2018 \\ https://doi.org/10.4193/Rhino17.189 \\ ' Department of Otorhinolaryngology, Division of Dentistry, Medical University of Warsaw, Warsaw, Poland \\ *Received for publication: \\ Department of Anatomy, University Medical Center Utrecht, Utrecht University, Utrecht, The Netherlands \\ August 19, 2017 \\ 3 Em. Professor of Otorhinolaryngology, University Medical Center Utrecht, Utrecht University, Utrecht, The Netherlands \\ Accepted: November 9, 2017
}

\begin{abstract}
The periosteum of the nasal bones, the periosteal-perichondrial nasal envelope, and the cartilaginous support of the bony vault were studied in serial coronal sections of four human cadaver noses. To differentiate between the various tissue components, the sections were stained according to Mallory-Cason and Verhoeff-Van Gieson stain. The results demonstrated: 1. the presence of clearly distinguishable layers of the periosteum covering the nasal bones; 2 . the presence of a continuous periosteal-perichondrial covering of the bony and cartilaginous nasal vaults; 3 . the way the cartilaginous support of the bony vault is constructed. The findings described in the present study may have clinical relevance in nasal surgery.
\end{abstract}

Key words: nasal bones, periosteum, perichondrium, rhinoplasty, septum

\section{Introduction}

The human external nose is an intricate anatomical-physiological structure. It is made out of various bony and cartilaginous structures, their periosteum and perichondrium, connective tissue with vessels and nerves, muscles, fatty tissue and skin. The nasal bones provide a rigid protective and supporting framework. The cartilages occur in different forms and provide structural support, as well as permitting varying degrees of mobility.

In previous articles we have reported and discussed our microscopic studies on the septal and lobular cartilages and their perichondrial envelope ${ }^{(1,2)}$. These structures are formed from highly specialized types of supporting connective tissue. The tissues are made up of an extracellular matrix, as well as the cellular elements that synthesise it. Questions regarding other aspects of the microscopic anatomy of the nose remain, such as whether there is a form-function relationship in several tissues of the external nose, and if supporting structures exist that contribute to the stability of the nose and might thus be relevant for nasal surgery.

Therefore, in the present study we have microscopically investigated:

1. the periosteum of the nasal bones,
2. the periosteal-perichondrial nasal envelope, and

3. the cartilaginous support of the bony vault.

\section{Materials and methods}

The external nose from four male cadavers of Caucasian origin aged $58,68,75$, and 89 years were studied. The specimens were preserved and fixed in $4 \%$ buffered formaldehyde, decalcified with sodium formiat solution, and then dehydrated in increasing concentrations of alcohol and embedded in paraffin. Serial sections of $10,15,20,25 \mu \mathrm{m}$ thickness were cut in the coronal plane at intervals of 200 and $400 \mu \mathrm{m}$ and then mounted on glass slides and stained by:

1. Mallory-Cason trichrome staining which colors bone: orange; cartilage: varying shades of blue; muscle fibers: brownish red; collagen: intense blue; elastin: pale pink, pale yellow, or unstained.

2. Verhoeff-Van Gieson stain to distinguish between collagen and elastin fibers. With this staining collagen fibers stain pink to red, whereas elastin fibers stain intensely blue, grey to black.

\section{Results}

The periosteum of the nasal bones

Our findings demonstrate two different periosteal layers:

1. a periosteum overlying both nasal bones and 
A

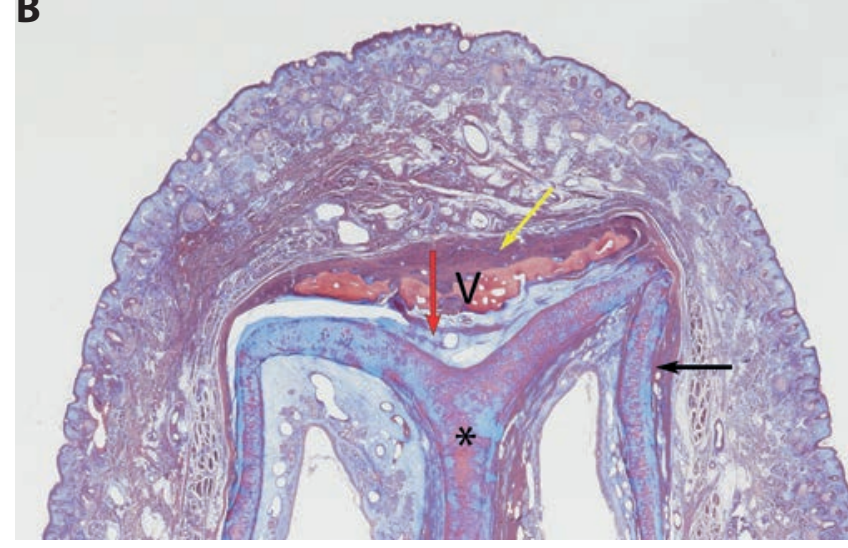

Figure 1. A) Overview of the external nasal pyramid showing a case of united nasal bones (arrowhead), the septolateral cartilage (asterisk) and adjacent structures (specimen 1, Mallory-Cason staining). B) Detail of A) with the two nasal bones grown together (arrowhead), their overlying periosteum (yellow arrow), the more or less triangular space under the nasal bones (red arrow), the septolateral cartilage (asterisk) with its perichondrium (black arrow) and the nasal soft tissues.

2. a periosteum that surrounds (covers) each nasal bone separately and fills the internasal suture. We suggest naming them the 'overlying periosteum' and the 'periosteum proper', respectively. The overlying periosteum is part of a periosteal-perichondrial envelope of the whole bony and cartilaginous nasal vault; the periosteum proper is the periosteum which surrounds the individual nasal bone (Figures 1a,b, 2a,b, 3a, b). The overlying periosteum consists of a rather thick layer of parallel running collagen fibres. There are no elastin fibers (Figure 4).

The 'periosteum proper' is thinner than the overlying periosteum and consists of other collagen fibers (Figures 2a, b). The internasal suture showed quite some variability. In one case the nasal bones had fused into one bony structure (Figures $1 a, b)$.

\section{The periosteal-perichondrial nasal envelope}

Our findings demonstrate that the thick overlying periosteum of the bony vault is continuous with the perichondrium of the triangular cartilages, sesamoid cartilages, and lobular cartilages; it envelops both the bony and cartilaginous vault (Figures 5a,

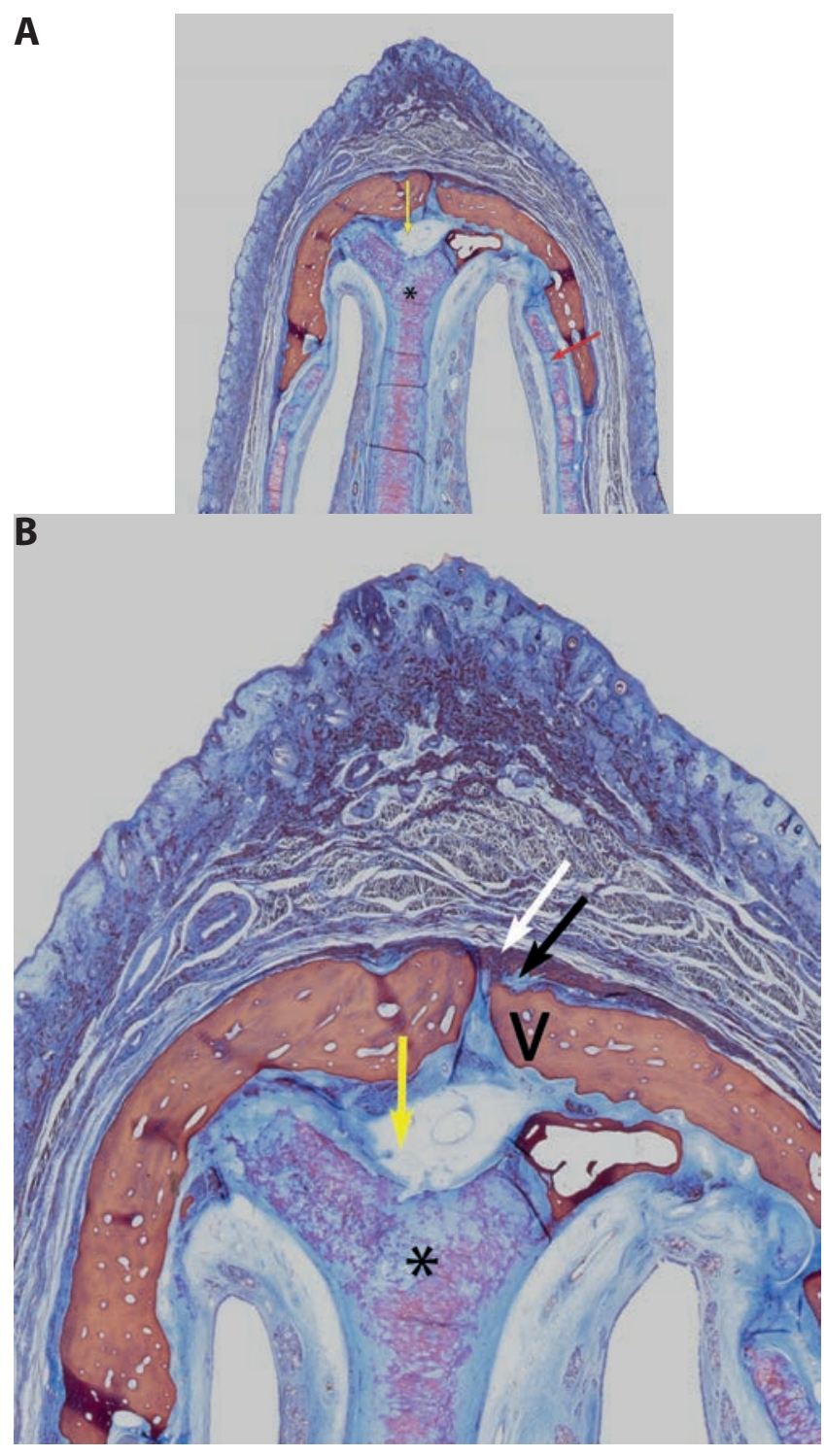

Figure 2. A) Overview of the nasal bones and adjacent structures. Note the underlay of the triangular cartilages (red arrow) under the nasal bones, the subnasal triangular space (yellow arrow) and the septal cartilage resembling the shape of a Y-beam (asterisk) (specimen 2, MalloryCason staining). B) Detail of A showing a continuous layer of the thick periosteum overlying both nasal bones (white arrow) and a thinner layer underneath, i.e. the periosteum proper (black arrow) which surrounds the individual nasal bone (arrowhead). The septal cartilage that supports the nasal vault has the shape of a Y-beam (asterisk). Between the septal cartilage and the nasal bones there is a somewhat triangular space (yellow arrow) that is filled with loose connective tissue and some blood vessels.

$b, 6 a, b)$. The coarse, closely packed bundles of collagen in the periosteal-perichondrial transition are interwoven by elastin fibers. These elastin fibers have a wave-like arrangement which may be related to the functional properties of the external nose (Figures 7a, b). 


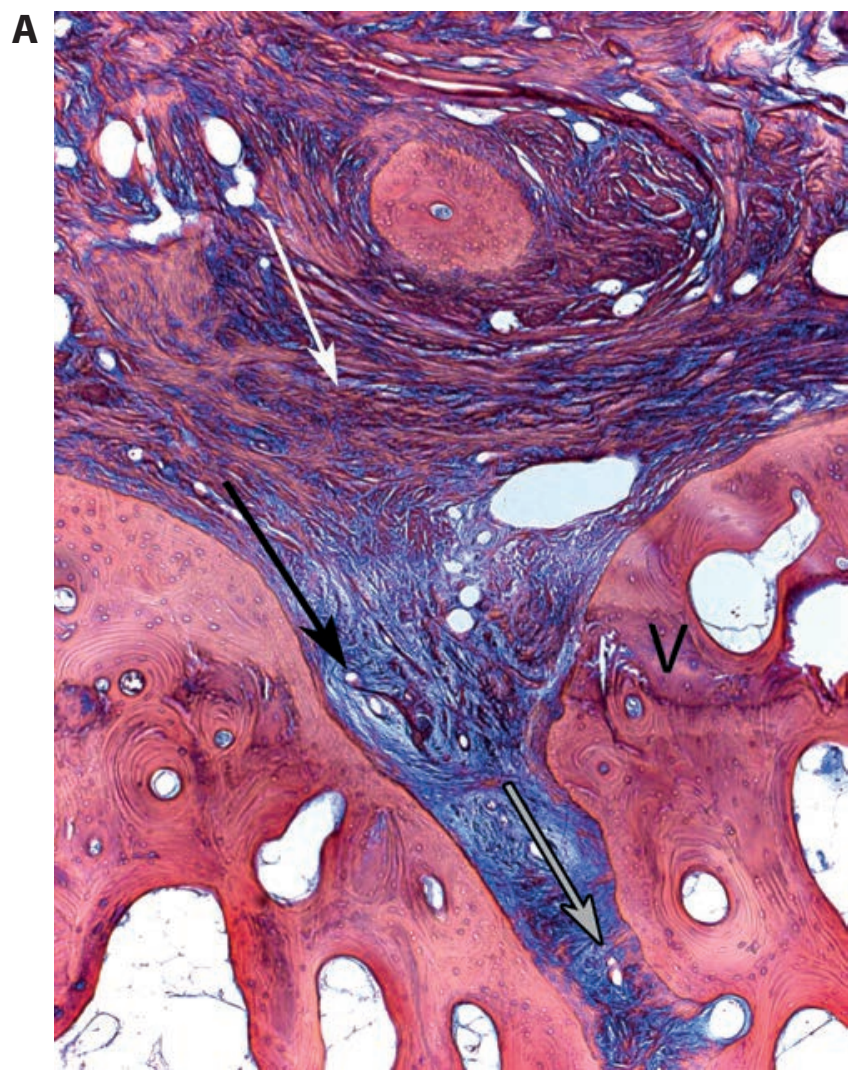

B

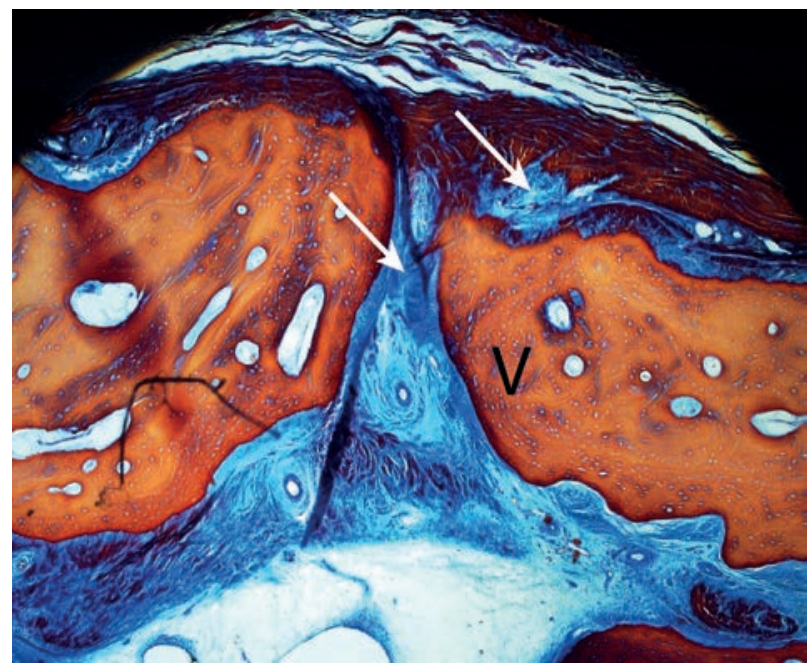

Figure 3. A) Higher magnification of the internasal suture and periosteum overlying the nasal bones. The thick layer of periosteum overlying both nasal bones contains collagen fibers (white arrow); the thin layer, i.e. the periosteum proper (black arrow), is composed of other collagen fibers running into the internasal suture (grey arrow), surrounding the individual nasal bone (arrowhead). In this specimen the two nasal bones have partially grown together (5x) (specimen 1, Mallory-Cason staining). B) Higher magnification of the internasal suture and the periosteum overlying the nasal bones (arrowhead). The internasal suture predominantly consists of the thin layer of the periosteum proper (white arrow) (5x) (specimen 2, Mallory-Cason staining).

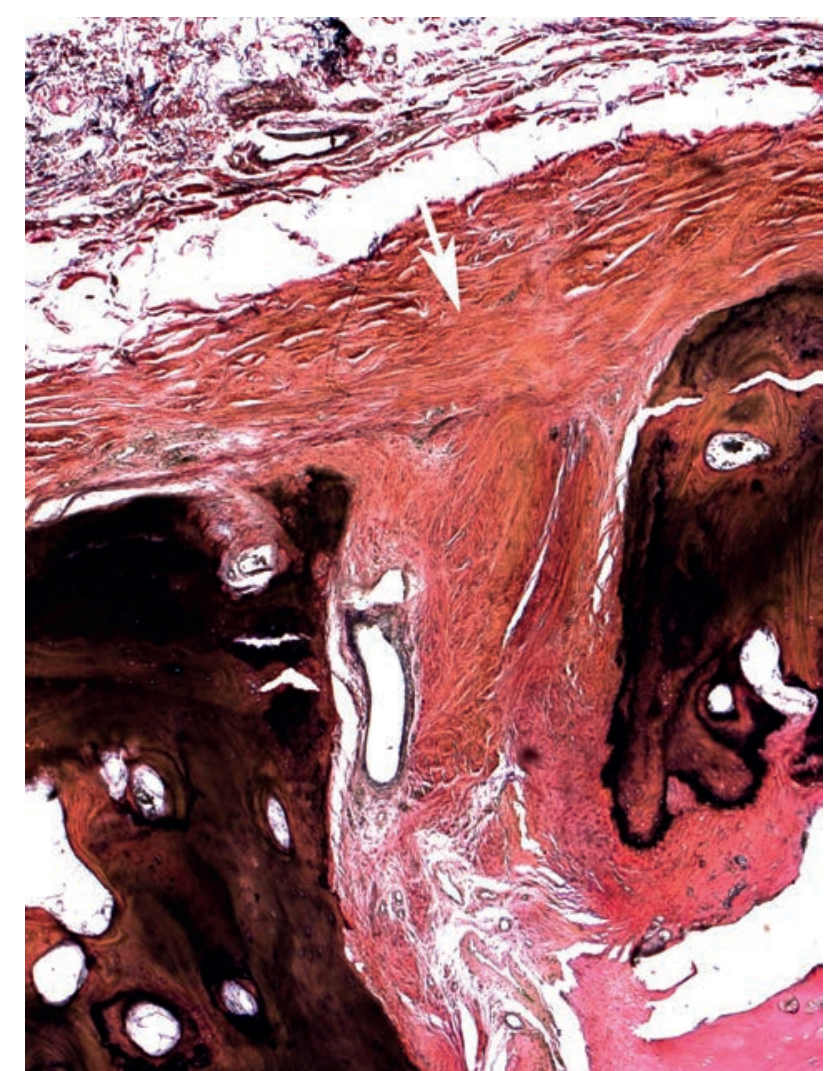

Figure 4. Higher magnification of the periosteum overlying the nasal bones (white arrow). With Verhoeff-van Gieson staining, collagen fibers of the periosteum stain pink to red, whereas elastin fibers stain intensely blue, grey to black. There are no elastin fibres present in the overlying periosteum (5x) (specimen 4, Verhoeff-van Gieson staining).

The cartilaginous support of the bony vault

In our study, we found that the following structures are involved in supporting the bony vault:

1. the Y-beam of the septal cartilage,

2. the resulting subnasal triangular space, and

3. the underlay of the triangular cartilages under the nasal bones.

The caudal part of the bony vault is supported by the septal cartilage that in this area is shaped like the letter Y. We propose naming this structure the septal Y-beam (Figures 1a,b, and 2a,b).

Between the two prongs of the septal Y-beam there is a somewhat triangular space that is filled with loose connective tissue and prominent vessels (Figures $8 a, b$ ). We suggest naming this area 'the subnasal triangular space'. Small and medium-sized vessels in the subnasal triangular space are arteries and veins. Some of the smaller vessels show recognizable features of arteries, i.e. the walls of arteries are thicker and more compact than those of veins. Among them, we observe a rather large artery 


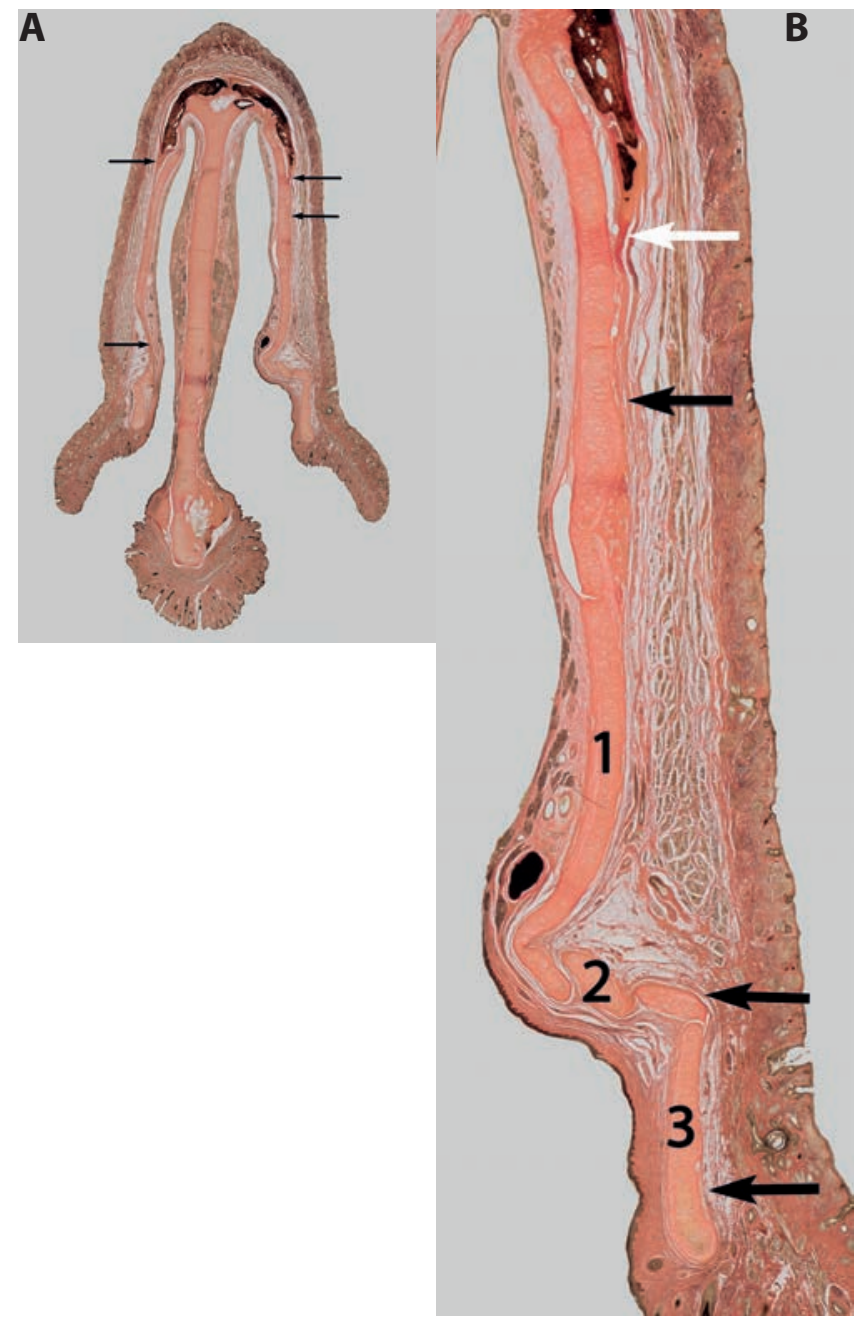

Figure 5. A) Overview of the external nasal pyramid with its periostealperichondrial covering (black arrows) of the bony and cartilaginous nasal vault. B) Detail of A demonstrating the thick periosteum (white arrow) of the nasal bones continuous with the perichondrium (black arrows) of the triangular cartilage (number 1), sesamoid cartilages (number 2), and lobular cartilage (number 3) (specimen 2, Verhoeff-van Gieson staining).

(white arrow) and vein (black arrow).

The bony and cartilaginous vault is characterized by an underlay of the cranial end of the triangular cartilage under the nasal bones (Figures 2a, 5a, and 7a). The bony and cartilaginous structures are linked to each other by the dense connective tissue that we above proposed naming 'the periosteal-perichondrial nasal envelope'.

\section{Discussion}

The main findings in this anatomical study are: 1. the presence of clearly distinguishable layers of the periosteum covering the nasal bones; 2 . the presence of a continuous periostealperichondrial covering of the bony and cartilaginous nasal
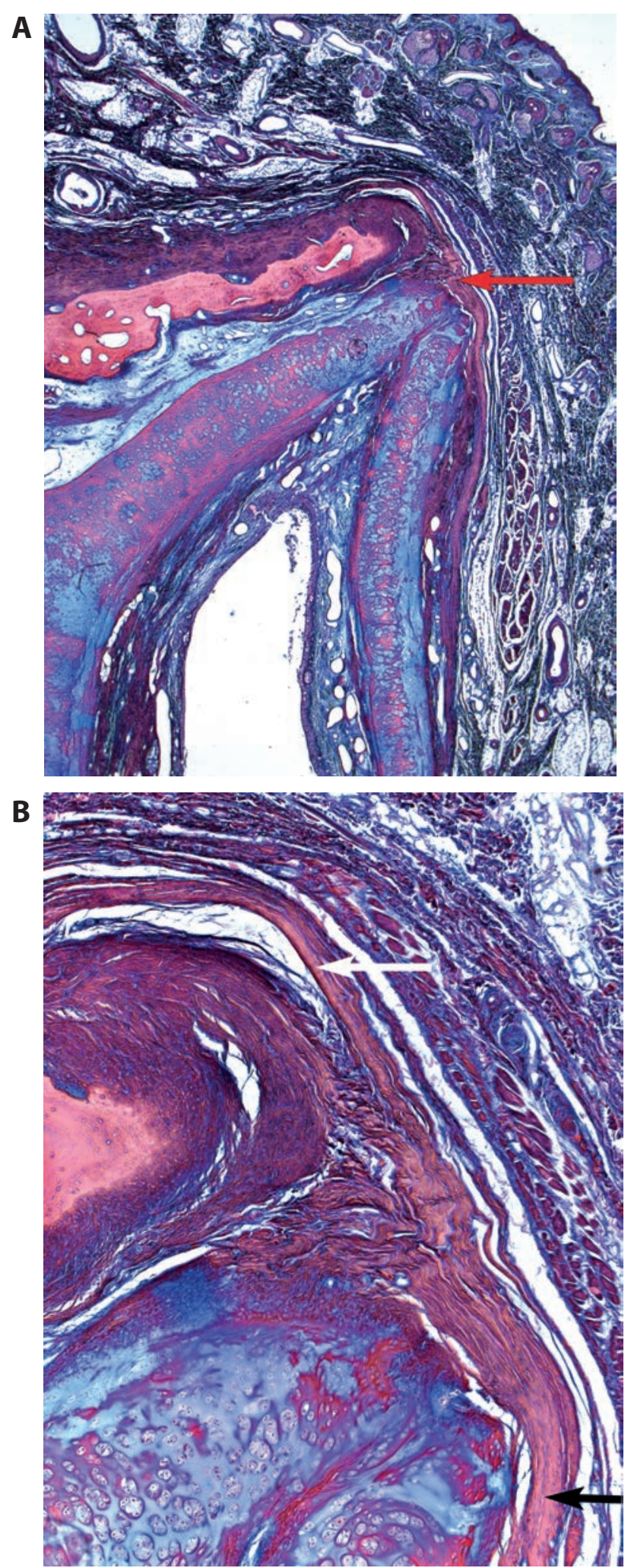

Figure 6. A) High magnification of a detail of Figure 1 showing the periosteal-perichondrial covering (red arrow) of the bony and cartilaginous nasal vault $(1,25 \mathrm{x})$. B) Higher magnification of a detail of Figure 1 showing the thick periosteum (white arrow) of the nasal bone continuous with the perichondrium (black arrow) of the triangular cartilage $(5 \mathrm{x})$ (specimen 1, Mallory-Cason staining). 
A

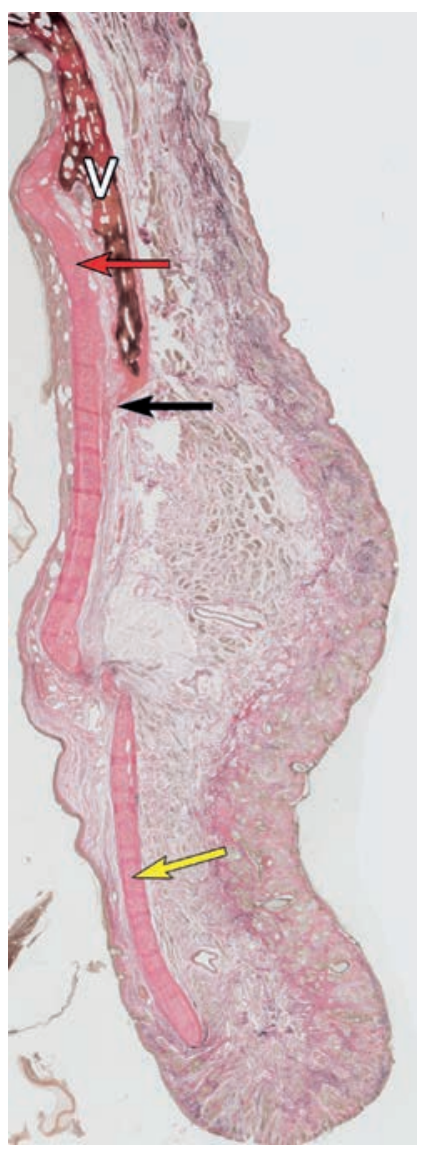

B

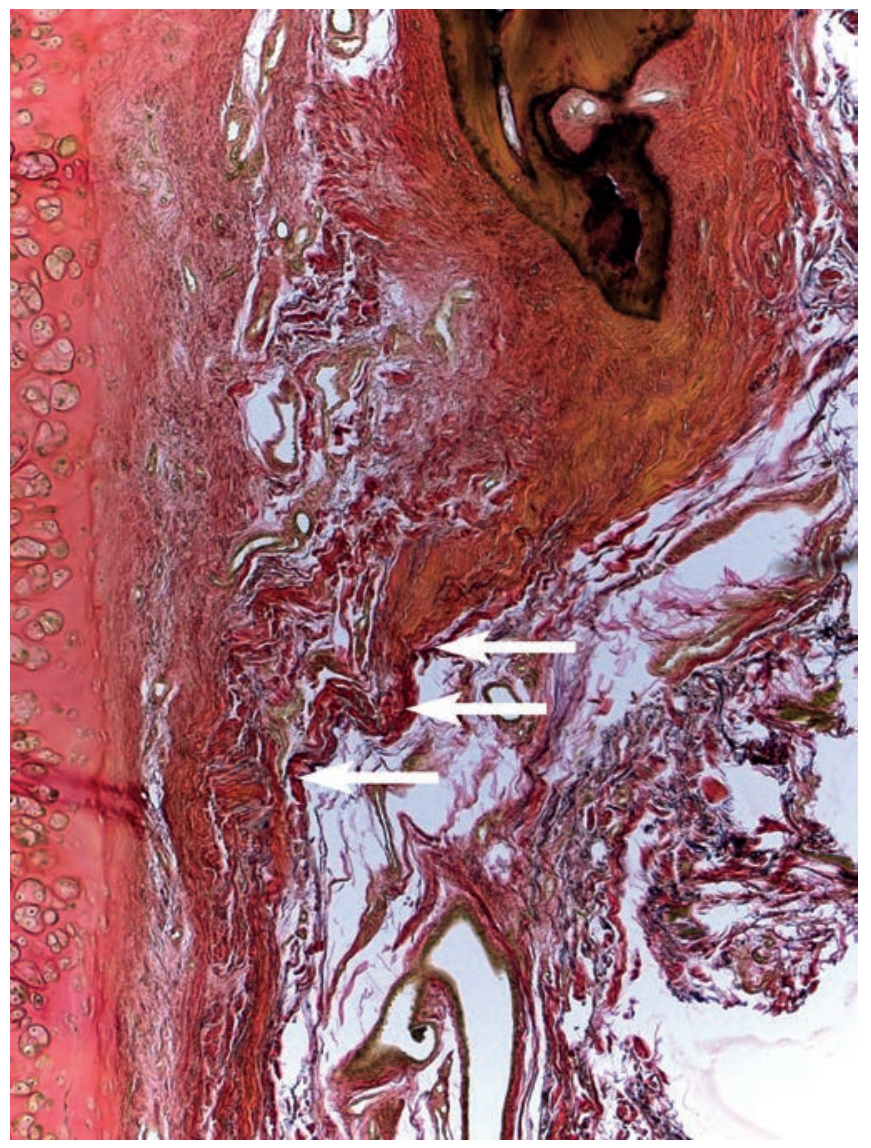

Figure 7. A) Overview of the lateral wall of the external nasal pyramid showing the relation (an underlay) of the triangular cartilage (red arrow) and the nasal bone (arrowhead), the lobular cartilage (yellow arrow), and the periosteal-perichondrial covering (black arrow) of the bony and cartilaginous nasal vault. B) High magnification of a detail of A) showing the periosteal-perichondrial covering of the bony and cartilaginous nasal vault at the underlay of the triangular cartilage and the nasal bone. The pink to red-stained, coarse, closely packed bundles of collagen in the periosteal-perichondrial transition are interwoven by elastin fibers (white arrows) that are stained black (5x) (specimen 4, Verhoeff-van Gieson staining).

vaults; 3 . the way the cartilaginous support of the bony vault is constructed.

Ad 1. One of the issues that we addressed was whether the structure of the periosteum of the nasal bones relates to its function. At the microscopic level we found that the periosteum of the nasal bones is composed of two layers: a thick layer of collagen fibers overlying both nasal bones, and a thinner layer of collagen fibers, i.e. the periosteum proper, surrounding the individual nasal bone. Our findings are consistent with previous reports that the periosteum covering the outside of bone is divided into two layers: a fibrous layer containing fibroblasts that produce type I collagen, and a cambial/osteogenic layer containing progenitor cells that can differentiate into osteoblasts that are responsible for increasing the thickness of bones $(3,4)$. Consistent with this knowledge, the overlying periosteum is responsible for the toughness of the whole periosteum. In corrective nasal surgery, the anatomic knowledge of nasal bones and its periosteum is of great importance ${ }^{(5)}$. Resecting a bony hump should be preceded by careful incision of the overlying periosteum in a vertical direction followed by scraping it aside to prevent its damage ${ }^{(6)}$. In reconstructing a saddle nose, on the other hand, a subperiosteal tunnel may thus be created preceding augmentation of the pyramid by dorsal transplants. Such a subperosteal tunnel is created between the overlying periosteum and the periosteum proper. The periosteum proper is the thin layer which surrounds each nasal bone. The periosteum proper plays a significant role in the growth, healing, and regeneration process of bone. The periosteum proper may thus have the capacity to repair after damage and contribute to the internasal suture. In rhinoplastic surgery, a medial (or more precisely a paramedian osteotomy) is therefore a fibrotomy instead of an osteotomy. In later life the connective tissue in the suture may ossify and as a consequence the two nasal bones become one bony structure.

Ad 2. The following item of focus was our finding of the continuous periosteal-perichondrial covering of the bony and 

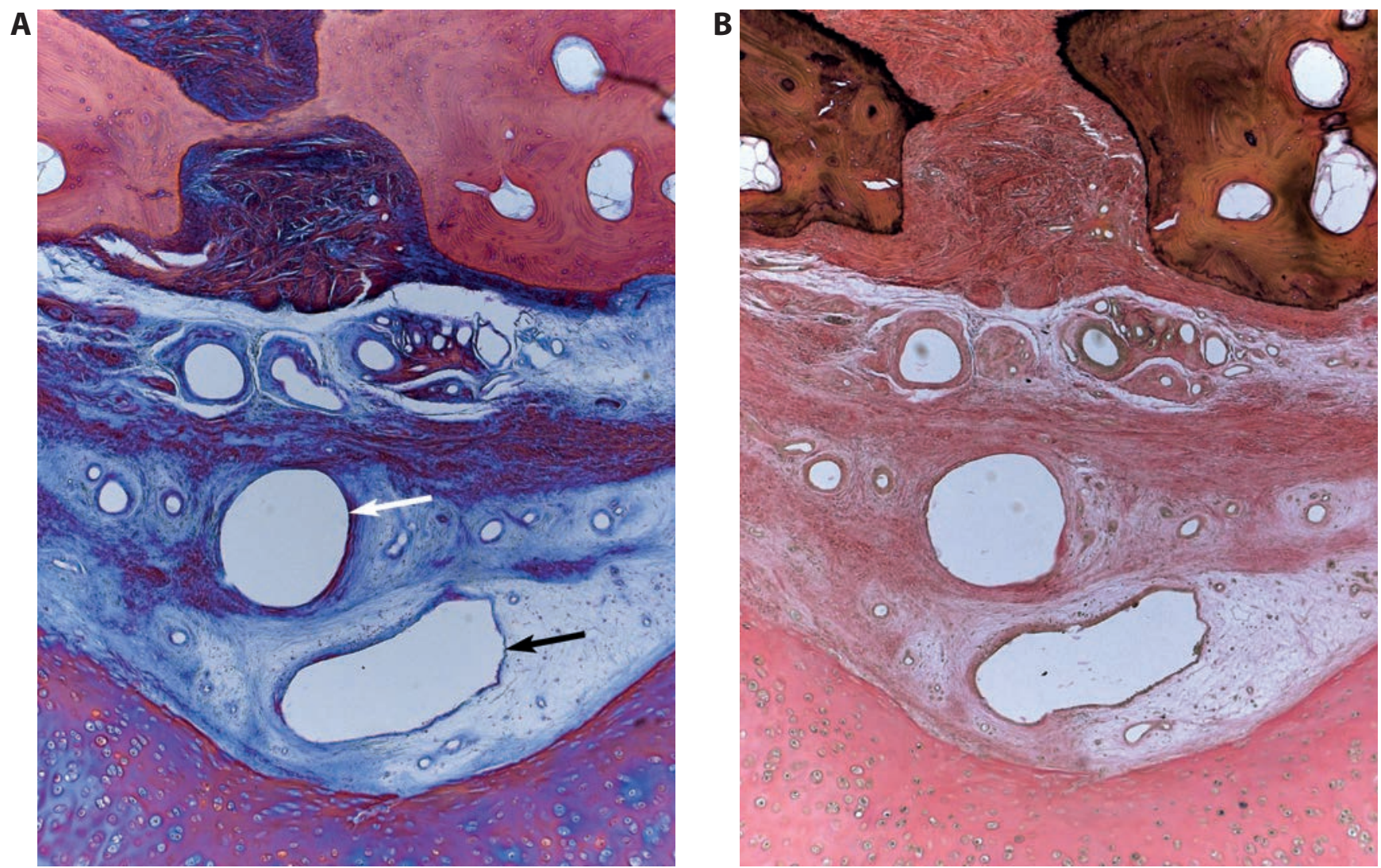

Figure 8. A) Higher magnification of the subnasal triangular space. The subnasal triangular space is composed of collagen fibers interspersed with blood vessels: artery (white arrow) and vein (black arrow) (5x) (specimen 1, Mallory-Cason staining). B) Higher magnification of the subnasal triangular space stained with Verhoeff-van Gieson stain shows an abundance of collagen fibers (staining red). There are no elastin fibers that would stain black with this method (5x) (specimen 1, Verhoeff-van Gieson staining).

cartilaginous nasal vaults. There have been numerous macroscopic and microscopic studies of the nasal soft tissue supporting structures. These studies have shown a fibrous connection between the lateral crus of the lobular cartilage, the triangular cartilage, and the piriform aperture ${ }^{(7-10)}$. In our study, we report the presence of a periosteal-perichondrial covering of the nasal bones and cartilaginous vault which additionally may contribute to the integrity and stability of the external nose. Disruption of the periosteal-perichondrial covering especially at the underlay between the triangular cartilage and the bony nasal pyramid may thus compromise the stability of the entire lateral nasal wall. During traditional rhinoplasty techniques, if the bony and cartilaginous dorsum is lowered, the triangular cartilages are intraseptally separated from the septum and thus dependent on their connection ${ }^{(11)}$, i.e. periosteal-perichondrial covering, with nasal bones for their support. Therefore the integrity of the periosteal-perichondrial covering should be protected by gentle and judicious dissection.

Ad 3. An interesting finding was the shape of the septal cartilage in the subnasal triangular space, the area where the septum un- derlies the nasal bones. There the shape of the septal cartilage resembles the letter $Y$. We propose naming this construction the septal Y-beam. This issue has not been recognized previously. Interestingly, originally the Y-beam model was developed in contemporary structural engineering for construction of bridges (12). The result of the parameter study, and requirements for a beam designed specifically for spaced beam and slab construction to adjust loading standards, led to the development of the new shape, the Y-beam. In this regard, it is interesting to note that the reason for the particular shape of the septal cartilage reflects its function, i.e. providing the best structural efficiency and robustness against collapse of the external nose.

While investigating the Y-beam and subnasal triangular space, we observed a robust blood supply in the latter. In general, mature cartilage has a very limited capacity to repair and regenerate, partly because of its poor blood supply. Most cartilage is devoid of blood vessels, and consequently the exchange of metabolites between chondrocytes and the surrounding tissues depends on diffusion through the water of the ground substance ${ }^{(3)}$. Our study showed that at the site where the cartilage particularly supports the nasal pyramid, a subnasal 
triangular space conveys prominent vessels into the region with a crucial supportive function. Consistent with the knowledge in histology ${ }^{(3)}$, in the subnasal triangular space we were able to distinguish several arteries of different size, and veins, when they run together. In corrective nasal surgery, resection in the region of the subnasal triangular space may damage vessels which could compromise the blood supply and limit the healing capacity. Given the complexity of the subnasal triangular space, it is essential to state that hump resection is rather a destructive procedure in rhinoplasty. Concerns for soft tissue and cartilage damage from hump removal stimulated surgeons to use power tools in this region of the nose ${ }^{(13,14)}$. Nevertheless, hump reduction is a persistent problem for many surgeons with relatively high number of complications. In cases where resection of the cartilaginous and bony part is necessary, one should keep in mind that it is possible to reduce the dorsum by bilateral wedge resection ${ }^{(6)}$.

The strength of this study is that we made sections of the entire external nose. This approach enabled us to study the continuity of all anatomical structures. A drawback of this approach is that it is technically challenging and labour-intensive. This is related to the limitation of the this study that only four nasal specimens were studied. However, given the consistency of the findings there is no reason to believe that increasing the number of specimens would lead to different results. This is in line with the literature which shows that many descriptive anatomical studies are based on a limited number of specimens ${ }^{(15,16)}$.

\section{Conclusion}

The detailed findings described in the present study may have clinical relevance in nasal surgery. When addressing the bony pyramid deformity, a subperiosteal tunnel is in fact created under the thicker layer of the periosteum overlying both nasal bones. Furthermore, avoiding disruption of the periostealperichondrial nasal envelope and preserving the cartilaginous support - i.e. the Y-beam - of the bony vault may maintain the stability of the external nose. Finally, preservation of the subnasal triangular space may reassure both the integrity of the cartilaginous support of the bony vault, and appropriate a blood supply crucial for the healing process. Understanding these findings provides new insight into the functional anatomy of the nose and thus may influence operating techniques.

\section{Acknowledgement}

We thank Marcel G.J. Nederhoff, Jan-Willem De Groot and Simon Plomp for their technical assistance.

\section{Authorship contribution}

Conceived and designed the experiments: EHH, RLAWB.

Performed the experiments: MP, SAMWVS. Analyzed the data:

EHH, RLAWB, MP. Contributed reagents/materials/analysis tools: RLAWB, SAMWVS. Wrote the paper: MP, EHH, RLAWB.

\section{Conflict of interest}

The authors declare no conflict of interest.

\section{References}

1. Popko M, Bleys RLAW, De Groot JW, Huizing EH. Histological structure of the nasal cartilages and their perichondrial envelope. I. The septal and lobular cartilage. Rhinology. 2007;45(2):148-52.

2. Bleys RLAW, Popko M, De Groot JW, Huizing EH. Histological structure of the nasal cartilages and their perichondrial envelope. II. The perichondrial envelope of the septal and lobular cartilage. Rhinology. 2007;45(2):153-7.

3. Young B, O'Dowd G, Woodford P. Wheater's Functional Histology: A Text and Colour Atlas. 6th ed. Elsevier, 2014.

4. Li N, Song J, Zhu G, Li X, Liu L, Shi X, et al. Periosteum tissue engineering-a review. Biomater Sci. 2016;4(11):1554-61.

5. Koppe T, Giotakis El, Heppt W. Functional anatomy of the nose. Facial Plast Surg. 2011;27(2):135-45.

6. Huizing EH, deGroot JAM. Functional reconstructive nasal surgery. 2nd ed. Thieme, 2015.

7. Janeke JB, Wright WK. Studies on the support of the nasal tip. Arch Otolaryngol. 1971:93(5):458-64.
8. Kridel RW, Konior RJ, Shumrick KA, Wright WK. Advances in nasal tip surgery. The lateral crural steal. Arch Otolaryngol Head Neck Surg. 1989;115(10):1206-12.

9. Dion MC, Jafek BW, Tobin CE. The anatomy of the nose. External support. Arch Otolaryngol. 1978;104(3):145-50.

10. Adamson PA, Morrow TA. The nasal hinge. Otolaryngol Head Neck Surg. 1994;111 (3 Pt 1):219-31.

11. Oneal RM, Beil RJ. Surgical anatomy of the nose. Clin Plast Surg. 2010;37(2):191-211.

12. Taylor HPJ, Clark LA, Banks CC. The Y-beam: a replacement for the $\mathrm{M}$-beam in beam and slab bridge decks. The Structural Engineer. 1990;68 (23/4):459-65

13. Gruber RP, Gupta D. Commentary on: The Osseocartilaginous Vault of the Nose: Anatomy and Surgical Observations. Aesthet Surg J. 2015;35(3):252-4.

14. Palhazi P, Daniel RK, Kosins AM. The osseocartilaginous vault of the nose: anatomy and surgical observations. Aesthet Surg J. 2015;35(3):242-51

15. Oomen KP, Ebbeling M, de Ru JA, Hordijk GJ, Bleys RLAW. A previously undescribed branch of the pterygopalatine ganglion.
Am J Rhinol Allergy. 2011;25(1):50-3.

16. Kraima AC, West NP, Treanor D, Magee DR, Rutten HJ, Quirke P, et al. Whole mount microscopic sections reveal that Denonvilliers' fascia is one entity and adherent to the mesorectal fascia; implications for the anterior plane in total mesorectal excision? Eur J Surg Oncol. 2015;41(6):73845.

Mariola Popko, MD, PhD

Otorhinolaryngology Department

Division of Dentistry

Medical University of Warsaw

19/25 Stepinska Str.

Warsaw

Poland

Tel: +48 694012224

Fax: +48 223186266

E-mail: popkom@interia.pl 